\title{
Inhibition of heat shock protein 90 alleviates cholestatic liver injury by decreasing IL-1 $\beta$ and IL-18 expression
}

\author{
CHENHAO TONG ${ }^{1 *}$, JIANDONG LI $^{1 *}$, WEIGUO LIN ${ }^{1,2}$, WENDA CEN $^{3}$, \\ WEIGUANG ZHANG ${ }^{4}$, ZHIYANG ZHU ${ }^{1}$, BAOCHUN LU ${ }^{1}$ and JIANHUA YU ${ }^{1}$ \\ ${ }^{1}$ Department of Hepatobiliary Surgery, Shaoxing People's Hospital (Shaoxing Hospital, \\ Zhejiang University School of Medicine), Shaoxing, Zhejiang 312000; ${ }^{2}$ Department of Urinary Surgery, \\ Ruian People's Hospital, Wenzhou, Zhejiang 325200; ${ }^{3}$ Department of Hepatobiliary Surgery, \\ The First Affiliated Hospital, Shaoxing University School of Medicine; ${ }^{4}$ Department of Molecular Medicine and \\ Clinical Laboratory, Shaoxing Second Hospital, Shaoxing, Zhejiang 312000, P.R. China
}

Received May 26, 2020 Accepted December 15, 2020

DOI: $10.3892 /$ etm.2021.9672

\begin{abstract}
Severe cholestatic liver injury diseases, such as obstructive jaundice and the subsequent acute obstructive cholangitis, are induced by biliary tract occlusion. Heat shock protein 90 (HSP90) inhibitors have been demonstrated to be protective for various organs. The potential of HSP90 inhibitors in the treatment of cholestatic liver injury, however, remains unclear. In the present study, rat models of bile duct ligation (BDL) were established, the HSP90 inhibitor 17-dimethylamino-ethylamino-17-demethoxygeldanamycin (17-DMAG) was administered, and its ability to ameliorate the cholestasis-induced liver injuries was evaluated. In the BDL rat models and clinical samples, increased HSP90 expression was observed to be associated with cholestatic liver injury. Furthermore, 17-DMAG alleviated cholestasis-induced liver injury in the rat models, as revealed by the assessment of pathological changes and liver function. In addition, 17-DMAG protected hepatocytes against cholestatic injury in vitro. Further assays indicated that $17-\mathrm{DMAG}$ administration prevented cholestasis-induced liver injury in the rats by decreasing the expression of interleukin (IL)-1 $\beta$ and IL-18. Moreover, 17-DMAG also decreased the cholestasis-induced upregulation of IL-1 $\beta$ and IL-18 in liver sinusoidal endothelial cells in vitro. In conclusion, the HSP90 inhibitor 17-DMAG is able to prevent liver injury in rats with biliary obstruction, and
\end{abstract}

Correspondence to: Dr Jianhua Yu or Dr Baochun Lu, Department of Hepatobiliary Surgery, Shaoxing People's Hospital (Shaoxing Hospital, Zhejiang University School of Medicine), 568 North Zhongxing Road, Shaoxing, Zhejiang 312000, P.R. China

E-mail: yujianhua@zju.edu.cn

E-mail: sygd_lbc@126.com

*Contributed equally

Key words: heat shock protein 90, 17-dimethylaminoethylamino-17-demethoxygeldanamycin, cholestasis, liver injury, interleukin- $1 \beta$, interleukin-18 this phenomenon is associated with the reduction of IL-1 $\beta$ and IL-18 expression.

\section{Introduction}

Obstructive jaundice is a frequently observed condition caused by biliary tract occlusion, which inevitably causes liver injury (1). In biliary obstruction, secondary gram-negative pathogen infection may occur, causing acute obstructive cholangitis, which progresses rapidly and has a poor prognosis $(2,3)$. The liver is the first organ to become involved and is severely injured during biliary obstruction. An important factor affecting prognosis is the extent of liver injury. Therefore, the identification of effective methods to alleviate cholestasis-induced liver injury can improve the prognosis of this disease.

Heat shock proteins (HSPs) are highly conserved proteins that are induced by a wide variety of physiological and environmental insults, such as heat, inflammation, toxic chemicals and oxidative stress. HSP90 is an essential member of the HSP family of chaperone proteins, and is upregulated in various conditions, including inflammation, organ injury and cancer (4-7). According to its subcellular localization, HSP90 comprises cytosolic HSP90 (HSP90AA1), endoplasmic reticulum-localized chaperone HSP90 (HSP90B1, also known as gp96 or grp94) and the mitochondrial member TRAP1 (HSP90L) (8). The potential therapeutic effects of HSP90 inhibitors, such as 17-dimethylamino-ethylamino-17-demethoxygeldanamycin (17-DMAG), have been the focus of a number of studies due to the important roles of HSP90 (9-11). Although studies have shown that 17-DMAG effectively prevents lipopolysaccharide (LPS)- or alcohol-induced liver injury $(11,12)$, the applicability of HSP90 inhibitors to biliary obstruction-induced liver injury remains unclear.

Toll-like receptors (TLRs) are a family of pattern recognition receptors for pathogens with critical roles in innate immunity (13). TLR9 is an essential TLR and regulates pro-inflammatory cytokines, such as interleukin (IL)-1 $\beta$ and IL-18 (14,15). In addition, HSP90B1 is required for the folding of TLR9 and is involved in the biogenesis of TLR9 $(16,17)$. 
TLR9 has been identified as a recipient protein of HSP90 and is associated with certain refractory diseases, such as hepatotoxicity (15).

In the present study, we hypothesized that HSP90 inhibition may reduce liver injury during biliary obstruction. Therefore, 17-DMAG was used to evaluate the effects of HSP90 inhibition in an animal model of biliary obstruction.

\section{Materials and methods}

Animal model. Wistar rats weighing 300-350 g and aged 12 weeks were purchased from Shanghai SLAC Laboratory Animal Co., Ltd. All procedures were approved by the Ethics Committee of Shaoxing People's Hospital and conformed to the Guide for the Care and Use of Laboratory Animals published by the US National Institutes of Health (revised 2011). The rats had ad libitum access to food and water and were maintained at $20^{\circ} \mathrm{C}$, with $50 \%$ humidity under 12 -h light/dark cycles.

Rats were randomly grouped into sham, bile duct ligation (BDL) and BDL + lipopolysaccharide (LPS) groups. The animals were anesthetized with an intraperitoneal injection of $50 \mathrm{mg} / \mathrm{kg}$ pentobarbital. In the BDL and BDL + LPS groups, the distal common bile ducts were dissociated and ligated with 6-0 silk, and PE-10 polyethylene catheters long enough to reach the skin were inserted into the proximal bile ducts as described in our previous study $(18,19)$. Intra-bile duct infusion was performed by injecting $0.2 \mathrm{ml}$ saline or LPS $(2 \mathrm{mg} / \mathrm{ml}$, purified from Escherichia coli O111:B4; Sigma-Aldrich; Merck KGaA) into the proximal bile ducts through the catheter. The catheter was sealed with a sealing cap and the abdominal cavity was covered using silk sutures following the introduction of $0.1 \mathrm{ml}$ air. The rats in the sham group underwent a sham procedure, which involved dissecting bile duct carefully without injury and closing the abdominal cavity. The rats in the sham, BDL and BDL + LPS groups were further divided into three groups according to the intraperitoneal injections they received after surgery. Briefly, in each group, one-third of the rats were intraperitoneally injected with normal saline (NS), another one-third were intraperitoneally injected with $2 \mathrm{mg} / \mathrm{kg}$ 17-DMAG (MedChemExpress) and the remaining one-third were intraperitoneally injected with $5 \mathrm{mg} / \mathrm{kg}$ 17-DMAG. The intraperitoneal injections were performed daily. A total of 108 rats were randomly classified into the aforementioned three groups according to the surgical procedure ( $\mathrm{n}=36 /$ group). Each of these groups comprised rats treated with NS, $2 \mathrm{mg} / \mathrm{kg}$ 17-DMAG and $5 \mathrm{mg} / \mathrm{kg}$ 17-DMAG ( $\mathrm{n}=12 /$ treatment), respectively. At 24 and $72 \mathrm{~h}$ after the surgery, a fraction ( $n=6 /$ group/time point) of the rats in each group were euthanized. Caspase- 3 activity detection and pathology experiments, including tissue staining and immunohistochemistry, were performed on samples taken at $72 \mathrm{~h}$. Other experiments were performed on samples taken at $24 \mathrm{~h}$. The rats were sacrificed via cervical dislocation under $2.5 \%$ sevoflurane inhalation anesthesia. The death of the animals was confirmed by examining the cessation of vital signs. Blood and liver tissue samples were harvested from the euthanized animals. Prior to analysis, blood samples were centrifuged $\left(1,000 \mathrm{xg}\right.$ for $5 \mathrm{~min}$ at $\left.4^{\circ} \mathrm{C}\right)$ and the serum was stored at $-80^{\circ} \mathrm{C}$. Liver tissues were snap-frozen in liquid nitrogen or fixed with $10 \%$ formalin.
Clinical tissue samples. Clinical tissue samples were obtained from patients who underwent hepatectomy at Shaoxing People's Hospital (Shaoxing, China) from January 2014 to December 2017. Normal liver tissues $(n=5$; age range, 42-63 years; inclusion criteria: Definite pathological diagnosis of hemangioma; exclusion criteria: Liver cirrhosis, fatty liver, hepatitis virus positive, liver tumor and hepatolithiasis) were obtained from patients who underwent hepatectomy to treat liver hemangioma; non-hemangioma tissues were selected for pathological examination. Intrahepatic cholangitic liver tissues $(n=5$; age range: $45-69$ years; inclusion criteria: Definite pathological diagnosis of hepatolithiasis; exclusion criteria: Liver cirrhosis, fatty liver, hepatitis virus positive and liver tumor, such as intrahepatic cholangiocarcinoma) were obtained from patients who underwent hepatectomy to treat intrahepatic stones and cholangitis. There were two male patients and three female patients in the two groups, respectively. All participants provided written informed consent, and Shaoxing People's Hospital Institutional Review Board approved the tissue acquisition protocol.

Evaluation of liver function. An Automated Chemical Analyzer (Dimension RxL Max HM; Siemens AG) was used to quantify the levels of aspartate aminotransferase (AST), alanine aminotransferase (ALT) and total bilirubin (TBIL) in rat serum to determine the degree of liver injury.

RNA extraction and reverse transcription-quantitative polymerase chain reaction ( $R T-q P C R)$. Total RNA was isolated from liver tissue samples or cells using TRIzol (Invitrogen; Thermo Fisher Scientific, Inc.) and reverse transcribed into cDNA using a PrimeScript ${ }^{\mathrm{TM}}$ RT reagent Kit (cat. no. RR047A; Takara Bio, Inc.), according to the manufacturer's protocols. Briefly, RT was conducted at $37^{\circ} \mathrm{C}$ for $15 \mathrm{~min}$ and $85^{\circ} \mathrm{C}$ for $5 \mathrm{sec}$, and the cDNA was stored at $4^{\circ} \mathrm{C}$ until further use. qPCR was performed using $\mathrm{SYBR}^{\circledR}$ Premix Ex Taq ${ }^{\mathrm{TM}}$ II (cat. no. RR820A; Takara Bio, Inc.) and an ABI 7500 Real-time PCR system (Applied Biosystems; Thermo Fisher Scientific, Inc.) according to the manufacturer's instructions. The primer sequences used are shown in Table I. $\beta$-actin was used as an endogenous control. The qPCR conditions comprised $95^{\circ} \mathrm{C}$ for $30 \mathrm{sec}$, and a total of 40 cycles at $95^{\circ} \mathrm{C}$ for $5 \mathrm{sec}$ and extension at $60^{\circ} \mathrm{C}$ for $34 \mathrm{sec}$, followed by a dissociation curve analysis. All assays were performed three times. Relative expression levels were then determined using the $2^{-\Delta \Delta \mathrm{Cq}}$ method (20).

Caspase-3 activity detection. A Caspase-3 Activity Assay Kit (cat. no. C1116; Beyotime Institute of Biotechnology) was used according to the manufacturer's protocol to detect the activity of caspase- 3 in the rat liver tissues. The absorbance was measured at $405 \mathrm{~nm}$ and the relative caspase-3 activity to the activity of the sham group was determined. Six samples from each group were analyzed.

Enzyme-linked immunosorbent assay (ELISA). The liver tissues were weighed and homogenized immediately in 10 volumes of saline after washing in saline. Supernatants were collected to perform ELISAs following centrifugation $\left(12,000 \mathrm{x} \mathrm{g}\right.$ for $10 \mathrm{~min}$ at $4^{\circ} \mathrm{C}$ ). ELISA kits (cat. nos. BMS630 and KRC2341, eBioscience; Thermo Fisher Scientific, Inc.) 
Table I. Primer sequences for quantitative polymerase chain reaction.

\begin{tabular}{|c|c|c|}
\hline Gene & Forward (5'-3') & Reverse (5'-3') \\
\hline$\beta$-actin & ACACCCGCCACCAGTTCG & CCCACGATGGAGGGGAAGA \\
\hline Hsp90aa1 & TCAGGCAGAAATTGCCCAGT & ATCCAGAGCGTCTGAGGAGT \\
\hline Hsp90b1 & ACCGAAAAGGACTTGCGACT & GCTCTCACAAACCCGAAGGT \\
\hline Trap1 & TGGCACCCGCAACATCTATT & CGTAGCAGAAGAGCACCTCA \\
\hline Hspa1b & CTCCTTCGTTCGGTCTGCAA & TGCAAAGACACACTCCCAGT \\
\hline Hspa4 & TCAGAGCTGCTATGTCGCTG & GGCATTGGAAATTACCTGGCTC \\
\hline Bax & GGGATGGCCTCCTTTCCTAC & CTTTCCCCGTTCCCCATTCA \\
\hline $\mathrm{Bcl} 2$ & AGCATGCGACCTCTGTTTGA & TCACTTGTGGCCCAGGTATG \\
\hline IL-1 $\beta$ & CAGCTTTCGACAGTGAGGAGA & TTGTCGAGATGCTGCTGTGA \\
\hline IL-18 & ACCGCAGTAATACGGAGCAT & CTGGGATTCGTTGGCTGTTC \\
\hline
\end{tabular}

Hsp90aa1, mitochondrial HSP90; Hsp90b1, cytosolic HSP90; Trap1, endoplasmic reticulum-localized HSP90; Hspa1b, HSP70 member 1b; Hspa4, HSP70 member 4; IL, interleukin.

were used according to the manufacturer's instructions to measure the content of IL-1 $\beta$ and IL-18 in the liver samples. Each sample was tested in triplicate.

Tissue staining. Liver tissue samples obtained from the rats were fixed with $10 \%$ formalin. The formalin-fixed specimens ( $3 \mu \mathrm{m}$ ) were stained using automatic H\&E slide stainer (Leica ST5020 Multistainer; Leica Microsystems, Inc.) according to the manufacturer's instructions for histological evaluation. The stained liver tissues were examined for histopathological evidence of pathological damage under light microscope (magnification, x100).

Immunohistochemistry (IHC). HSP90 expression in the rat liver tissues was detected by IHC. Deparaffinization and rehydration of the sections ( $3 \mu \mathrm{m}$ thickness) were performed with gradient xylene and a descending ethanol gradient. Antigen retrieval was performed with Citrate Antigen Retrieval solution (1:50; cat. no. P0083; Beyotime Institute of Biotechnology) at $95^{\circ} \mathrm{C}$ for $20 \mathrm{~min}$. Endogenous peroxidase activity was quenched using $0.3 \%$ hydrogen peroxide $\left(25^{\circ} \mathrm{C}, 10 \mathrm{~min}\right)$. The sections were blocked with $1 \%$ BSA (Beyotime Institute of Biotechnology) for $2 \mathrm{~h}$ at room temperature. The sections were then incubated with primary anti-HSP90 antibody $(1: 100$; cat. no. 13171-1-AP; ProteinTech Group, Inc.) overnight at $4^{\circ} \mathrm{C}$, followed by incubation with horseradish peroxidase-conjugated secondary antibody (1:50; cat. no. A0208; Beyotime Institute of Biotechnology) for $1.5 \mathrm{~h}$ at room temperature. After thoroughly washing with washing buffer (cat. no. P0106L; Beyotime Institute of Biotechnology), the sections were developed using a 3,3'-diaminobenzidine kit (cat. no. P0202; Beyotime Institute of Biotechnology) and counterstained with hematoxylin staining solution (cat. no. C0107; Beyotime Institute of Biotechnology) for $10 \mathrm{~min}$ at room temperature. Each stained sample was observed under high power magnification using a light microscope (magnification, x200; Leica Microsystems, Inc.).

'Cholestatic culture' assay. BDL and sham rat models were prepared using the aforementioned procedure. After 1 week, cholestatic blood samples were harvested from the rats and cholestatic serum was obtained after centrifugation $(2,000 \mathrm{xg}$ for $10 \mathrm{~min}$ at $4^{\circ} \mathrm{C}$ ). Cholestatic serum samples from all BDL model rats were mixed in one tube and non-cholestatic serum samples from all sham model rats were mixed in another tube. The sera were filtered using a $0.22-\mu \mathrm{m}$ filter (Millipore; Merck KGaA) for sterilization. The automated chemical analyzer was used to examine the cholestatic and non-cholestatic serum. The cholestatic serum contained $188.02 \mu \mathrm{mol} / 1$ total bilirubin (TBIL) and $146.6 \mu \mathrm{mol} / 1$ direct bilirubin (DBIL), while the non-cholestatic serum contained $2.3 \mu \mathrm{mol} / 1 \mathrm{TBIL}$ and $1.72 \mu \mathrm{mol} / 1 \mathrm{DBIL}$. The two types of serum were used in the subsequent experiments.

The BRL rat hepatic cell line was obtained from the Shanghai Cell Bank of the Chinese Academy of Sciences. Liver sinusoidal endothelial cells (LSECs) were isolated from the rat liver tissues with modifications to the previously described protocol (21). Briefly, the rat liver tissue was perfused with collagenase (Invitrogen; Thermo Fisher Scientific, Inc.). The single-cell suspension was subjected to velocity and density centrifugations $\left(50 \mathrm{x} \mathrm{g}\right.$ for $2 \mathrm{~min}$ at $\left.25^{\circ} \mathrm{C}\right)$ in Percoll gradients to separate hepatocyte and nonparenchymal cell suspensions. The nonparenchymal cell suspension was incubated with CD146 (LSEC)-biotin antibodies (1:50; cat. no. 130-111-844; Miltenyi Biotec, Inc.) for cell surface staining (30 min at room temperature). The LSEC cells were positively selected by magnetic separation using Anti-Biotin MicroBeads (Miltenyi Biotec, Inc.) according to the manufacturer's instructions.

Prior to stimulation with serum, BRL cells were cultured in Dulbecco's modified Eagle's medium (DMEM; Gibco; Thermo Fisher Scientific, Inc.) supplemented with $10 \%$ fetal bovine serum (FBS; Gibco; Thermo Fisher Scientific, Inc.), and LSECs were cultured with a medium comprising 40\% MCDB 131 (Gibco; Thermo Fisher Scientific, Inc.), 40\% RPMI-1640 (Gibco; Thermo Fisher Scientific, Inc.) and $20 \%$ FBS. The 'cholestatic culture' was initiated when the FBS was replaced with the cholestatic serum or non-cholestatic serum obtained from the rats.

Cell Counting Kit-8 (CCK-8) assay. BRL cells were seeded into a 96 -well plate at a density of $2 \times 10^{3}$ cells/well and allowed 
to attach overnight at $37^{\circ} \mathrm{C}$. The cells were then cultured in a medium containing non-cholestatic serum or cholestatic serum, with or without 17-DMAG $(0.5 \mu \mathrm{M})$ for $48 \mathrm{~h}$. Cell proliferation was determined using the CCK- 8 assay (MedChemExpress). Briefly, a 1/10 volume of CCK-8 solution was added to each well and the cells were cultured for $2 \mathrm{~h}$. Cell viability was then determined by measuring the absorbance at $450 \mathrm{~nm}$ using a plate reader (BioTek Instruments, Inc.). Six repeated wells for each cell condition were analyzed.

Flow cytometric analysis. BRL cells were cultured in a cholestatic medium with or without 17-DMAG $(0.5 \mu \mathrm{M})$ at $37^{\circ} \mathrm{C}$. After $24 \mathrm{~h}$, the cells were collected and stained using Annexin V-FITC and propidium iodide (Becton, Dickinson and Company) according to the manufacturer's instructions. Apoptotic cells were quantified by flow cytometry using a Flow Cytometer (Cytomics FC500; Beckman Coulter, Inc.). The data was analyzed with CXP software version 1.0 (Beckman Coulter). The experiments were performed in triplicates.

Statistical analysis. Data are presented as the mean \pm SD. The statistical significance of a difference between two groups was determined using the Student's t-test. Two-way ANOVA followed by Bonferroni's adjustment were used to evaluate differences among multiple groups. $\mathrm{P}<0.05$ was considered to indicate a statistically significant result. All statistical analyses were conducted using SPSS 13.0 software (SPSS, Inc.).

\section{Results}

HSP90 expression is upregulated during cholestatic liver injury. Serum levels of ALT, AST and TBIL were examined to confirm the successful establishment of the animal model. The significantly increased ALT, AST and TBIL levels in the BDL group compared with sham group were indicative of substantial cholestasis and liver injury in the BDL group (Fig. 1A). In addition to simple biliary obstruction, the gram-negative pathogen LPS is another factor that aggravates biliary obstruction-induced liver injury (22). Therefore, a BDL + LPS rat model was established to simulate frequently occurring clinical cases. As shown in Fig. 1A, LPS administration further aggravated the BDL-induced liver injury.

The mRNA expression levels of three HSP90 family members in the liver tissues of the BDL and BDL + LPS rat models were compared with those in the sham group to clarify the association between HSP90 expression and cholestatic liver injury. In the BDL and BDL + LPS groups, the results showed that Hsp90aal and Hsp90b1 mRNA levels were significantly upregulated compared with those in the sham group (Fig. 1B). IHC showed that the HSP90 protein expression was also upregulated in the liver tissues of rats in the BDL and BDL + LPS groups (Fig. 1C). Whether a similar phenomenon exists in human liver tissues was also examined. Liver specimens obtained from patients with hepatolithiasis or intrahepatic cholangitis who had undergone hepatectomy were analyzed using IHC. The results demonstrated that HSP90 expression was upregulated in the patients with intrahepatic cholangitis (Fig. 1D). These results imply that aberrant HSP90 expression is associated with cholestasis and cholangitis.
HSP90 inhibitor 17-DMAG alleviates cholestasis-induced liver injury. Previous studies have supported the potential of 17-DMAG, a water-soluble HSP90 inhibitor, as a therapeutic agent $(23,24)$. The effect of 17-DMAG on cholestasis-induced liver injury was therefore investigated in vivo to identify whether the inhibition of HSP90 enhances the transcription of HSP70, as indicated by previous studies (25-27). The elevated mRNA levels of HSP70 members $1 \mathrm{~b}$ and 4 (Hspa1b and Hspa4, respectively) in the rats following 17-DMAG administration indicated that the activity of HSP90 was effectively inhibited (Fig. 1E).

Following the administration of 17-DMAG or NS to the rats, the serum levels of ALT and AST were assessed. ALT and AST levels in the BDL and BDL + LPS groups exhibited significant reductions upon treatment with 17-DMAG compared with those in the NS-treated rats (Fig. 1A). However, 17-DMAG administration did not significantly mitigate the increase of TBIL levels after the onset of cholestasis (Fig. 1A).

A morphological assessment was performed to evaluate the degree of injury exhibited by the rat liver tissue. Pathological examination revealed dilation of the bile capillaries and increased local necrosis in the livers of rats with BDL-induced cholestasis (Fig. 1F). In addition, following LPS administration, large areas of necrosis and increased inflammatory cell infiltration were observed (Fig. 1F). However, compared with NS treatment, 17-DMAG treatment reduced local necrosis and inflammatory cell infiltration during cholestasis with or without LPS (Fig. 1F).

Caspase- 3 activity and the ratio of Bax to $\mathrm{Bcl} 2$ are often used to evaluate the apoptotic response (28). Notably, caspase-3 activity increased significantly in the liver tissues of the BDL and BDL + LPS groups compared with the sham group. However, treatment with 17-DMAG effectively attenuated this change (Fig. 1G). Furthermore, the Bax to $\mathrm{Bcl} 2$ ratio exhibited similar changes (Fig. 1H). These results indicate that 17-DMAG effectively alleviated cholestasis-induced liver injury.

17-DMAG protects hepatocytes against cholestasis-induced cell damage in vitro. A 'cholestatic culture' experiment was conducted to further clarify the function of 17-DMAG during cholestasis-induced cell damage in vitro. Cholestatic serum from BDL model rats was added to the culture medium of BRL cells to simulate the cholestatic microenvironment in vitro. CCK-8 assays were then performed to evaluate cell viability. Moderate cytotoxicity was observed when BRL cells were treated with cholestatic serum (Fig. 2A). However, 17-DMAG administration significantly alleviated this cytotoxicity, whether or not LPS was present in vitro (Fig. 2A). In addition, the flow cytometric analysis of cell apoptosis indicated that 17-DMAG administration promoted the survival of BRL cells and reduced the number of apoptotic cells in the cholestatic serum-containing medium (Fig. 2B).

17-DMAG administration prevents cholestasis-induced liver injury by decreasing the expression of $I L-1 \beta$ and $I L-18$ in vivo. Pro-inflammatory cytokines induced by bile acid play an important role in cholestasis-induced liver injury $(29,30)$. IL-1 $\beta$ and IL-18 are important cytokines, as they have a role in the induction of apoptosis during liver injury $(15,31)$. Therefore, they were analyzed in the present study. The results 

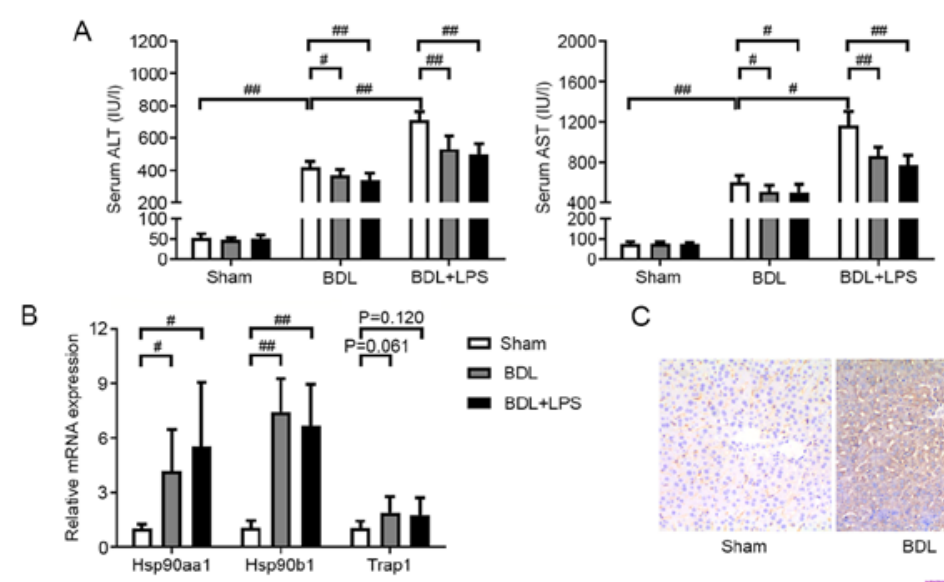

C


Figure 1. 17-DMAG alleviates cholestatic liver injury in vivo. (A) ALT, AST and TBIL were examined in rat serum $24 \mathrm{~h}$ after BDL or sham surgery (n=6/group). The rapidly increased ALT, AST and TBIL levels demonstrated that cholestasis-induced liver injury occurred in the BDL and BDL + LPS groups. (B) Relative expression of HSP90 mRNA in liver tissues from three rat models ( $\mathrm{n}=6 /$ group). Hsp90aa1 and Hsp90b1 mRNA were upregulated after biliary obstruction. (C) IHC staining shows that HSP90 protein was upregulated $72 \mathrm{~h}$ after biliary obstruction (images are representative of 6 rats/group; magnification, $\mathrm{x} 200$ ). (D) IHC staining shows that HSP90 expression was upregulated in patients with intrahepatic cholangitis compared with (images are representative of five rats/group; magnification, x200). (E) Relative mRNA expression levels of HSP70 family members Hspalb and Hspa4 in rat liver tissues (n=6/group). Elevated mRNA levels of HSP70 indicate that the activity of HSP90 was effectively inhibited. (F) Histological examination (magnification, x100), (G) the activity of caspase- 3 and $(\mathrm{H})$ the ratio of Bax to Bcl2 expression levels show that 17-DMAG alleviated cholestasis-induced liver injury (n=6/group). Black arrows indicate inflammatory cell infiltration and yellow arrows indicate necrosis. ${ }^{~} \mathrm{P}<0.05 ;{ }^{\# \#} \mathrm{P}<0.01$. 17-DMAG, 17-dimethylamino-ethylamino-17-demethoxygeldanamycin; ALT, alanine aminotransferase; AST, aspartate aminotransferase; TBIL, total bilirubin; NS, normal saline; HSP90, heat shock protein 90; Hsp90aa1, mitochondrial HSP90; Hsp90b1, cytosolic HSP90; Trap1, endoplasmic reticulum-localized HSP90; IHC, immunohistochemistry; BDL, bile duct ligation; LPS, lipopolysaccharide; HSP70, heat shock protein 70; Hspa1b, HSP70 member 1b; Hspa4, HSP70 member 4; casp3, caspase-3; n.s., not significant.

showed that the mRNA expression levels of IL-1 $\beta$ and IL-18 increased significantly during cholestasis-induced liver injury, and treatment with 17-DMAG substantially attenuated these changes (Fig. 3A). ELISAs of the liver tissues also demonstrated that the cholestasis-induced increases in IL-1 $\beta$ and IL-18 levels were significantly attenuated following treatment with 17-DMAG (Fig. 3B).

17-DMAG administration decreases the expression of IL-1 $\beta$ and IL-18 by LSECs in vitro. LSECs serve an important role in the immune response and liver injury $(32,33)$. LSECs were isolated from rats for in vitro analyses to study the underlying mechanism. The results showed that the addition of cholestatic medium significantly increased the expression of IL- $1 \beta$ and IL-18 by LSECs, compared with non-cholestatic medium (Fig. 3C and D). Notably, the administration of 17-DMAG significantly attenuated the increase in the expression of IL-1 $\beta$ and IL-18 induced by cholestatic medium (Fig. 3C and D).

Mammalian TLRs detect microbial infection and regulate the cell death-associated cellular response during stress. The HSP90 family, particularly HSP90B1, have been identified as TLR chaperones that inhibit TLR signaling and the biogenesis of downstream pro-inflammatory cytokines $(16,17)$. TLR9 has been reported to induce liver injury via the regulation of IL-1 $\beta$ and IL-18 (15,34). Therefore, the TLR9 inhibitor E6446 dihydrochloride was used to treat the LSECs. The administration of E6446 also reversed the cholestatic medium-induced expression of IL-1 $\beta$ and IL-18, in a comparable manner to 17-DMAG (Fig. 3C and D). These results suggest a potential association between the protective effect of HSP90 inhibitors on the liver and the regulation of pro-inflammatory cytokine biogenesis by TLR9. 

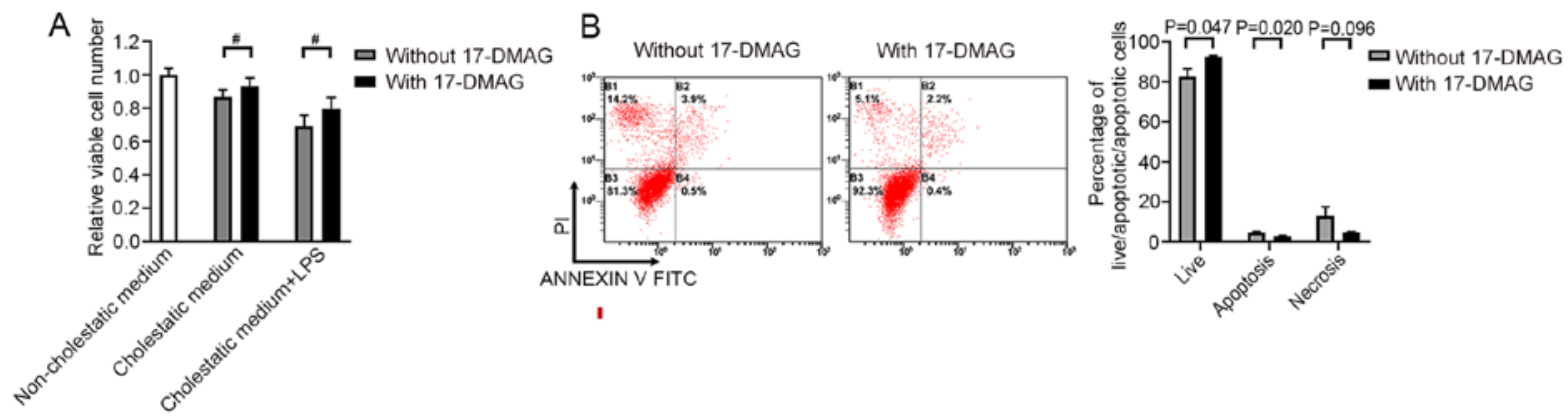

Figure 2. 17-DMAG promotes the survival of hepatocytes in vitro. (A) Cell viability assays $(n=6)$ and (B) flow cytometric analysis $(n=3)$ of BRL cells show that 17-DMAG protected hepatocytes against the damaging effect of culture with cholestatic serum samples from bile duct-ligated rats in vitro. ${ }^{\text {}} \mathrm{P}<0.05$. 17-DMAG, 17-dimethylamino-ethylamino-17-demethoxygeldanamycin; LPS, lipopolysaccharide.
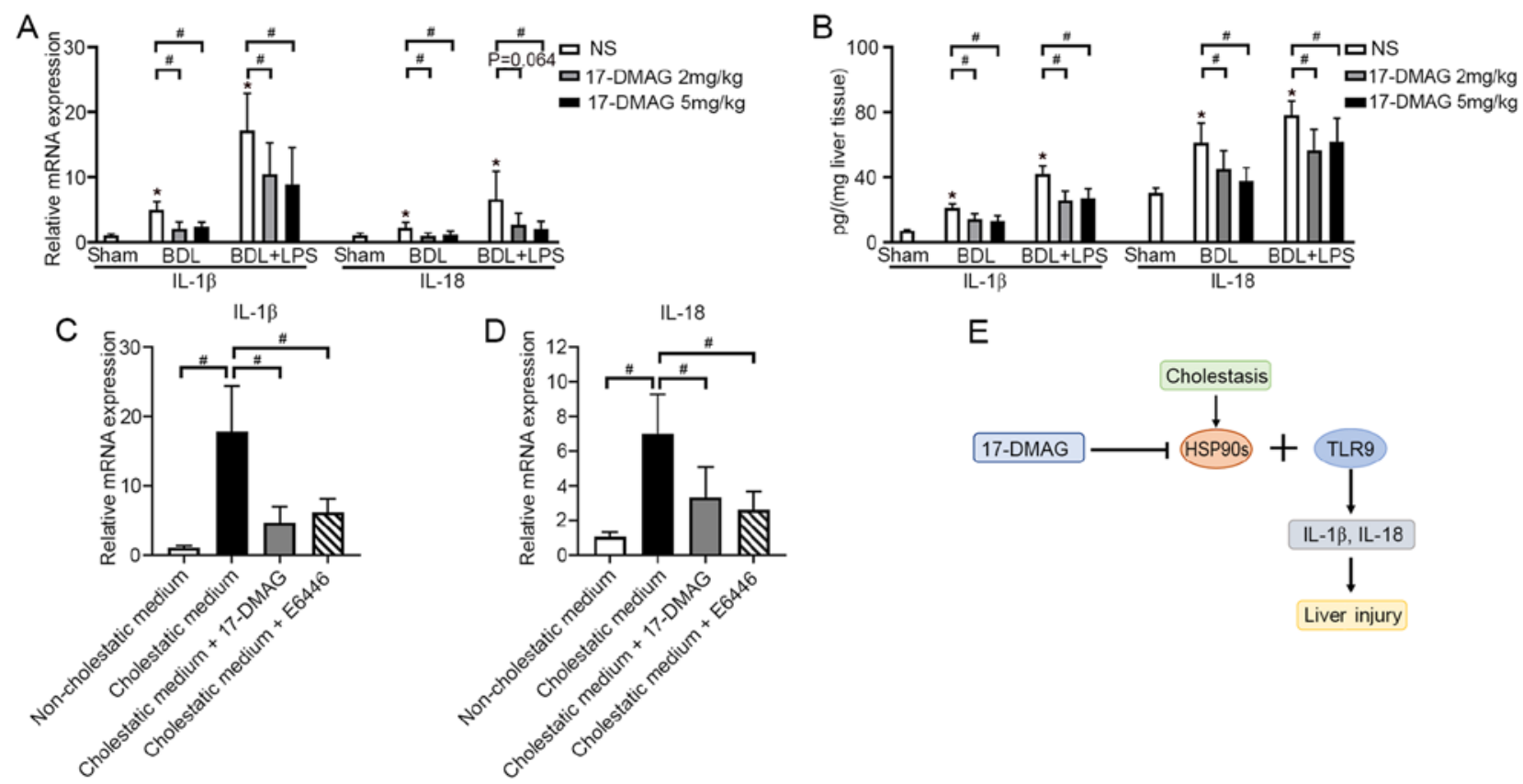

Figure 3. 17-DMAG inhibits IL-1 $\beta$ and IL-18 expression in the liver during cholestasis. (A) RT-qPCR assays and (B) ELISAs show that 17-DMAG diminished the cholestasis-induced increased expression of IL-1 $\beta$ and IL-18 in rat liver tissues ( $\mathrm{n}=6$ /group). RT-qPCR assays show that 17-DMAG and the TLR9 inhibitor E6446 independently decreased the cholestasis-induced expression of (C) IL-1 $\beta$ and (D) IL-18 in LSECs. Experiments were repeated three times. (E) Schematic representation of how HSP90 and TLR9 signaling regulates the expression of IL-1 $\beta$ and IL-18 during cholestasis. "P<0.05 vs. Sham group. ${ }^{\text {"P}} \mathrm{P}$ 0.05. 17-DMAG, 17-dimethylamino-ethylamino-17-demethoxygeldanamycin; IL, interleukin; RT-qPCR, reverse transcription-quantitative polymerase chain reaction; ELISA, enzyme-linked immunosorbent assay; LSECs, liver sinusoidal endothelial cells; HSP90, heat shock protein 90; TLR9, Toll-like receptor 9; BDL, bile duct ligation; LPS, lipopolysaccharide.

\section{Discussion}

Biliary obstruction is a common clinical symptom in various benign or malignant diseases and often causes severe liver injury. HSP90 inhibitors have been described as protective drugs for various organs due to their anti-inflammatory effects $(11,12,35-37)$. Given that the present study observed that HSP90 expression increased significantly in cholestatic human and rat livers, the inhibition of HSP90 may be a potential method for the alleviation of liver injury during biliary obstruction. Both simple biliary obstruction and biliary obstruction with bacterial infection cause liver injury in clinical cases. Given that gram-negative bacteria are the most common pathogens associated with biliary infection and LPS is the most important pathogenic factor of gram-negative bacteria, a BDL + LPS group was employed in the present study to simulate biliary obstruction with secondary infection, in addition to a BDL group. The results demonstrated that the HSP90 inhibitor 17-DMAG significantly decreased the levels of ALT and AST and alleviated the pathological changes caused by cholestasis with or without LPS. These results strongly imply that HSP90 inhibitors can protect the liver from cholestatic injury.

A novel cell experiment named 'cholestatic culture' was designed and performed in the present study to clarify the protective function of 17-DMAG against cholestasis-induced cell damage in vitro. In a preliminary experiment, the dissolution of bilirubin in DMEM was attempted in order to simulate the microenvironment of hepatocytes after biliary obstruction. Although the addition of dimethyl sulfoxide improved the solubility of bilirubin, this method only provided a culture medium containing unconjugated bilirubin. However, following biliary obstruction, the increase in serum bilirubin is often 
characterized by a sharp increase in conjugated bilirubin rather than unconjugated bilirubin (1). Therefore, since both BRL cells and LSECs are rat cells, cholestatic serum from BDL rats was used instead. Whether the rat serum influenced the growth of BRL cells in vitro was examined using CCK-8 assays. The BRL cells grew faster in medium supplemented with rat serum than in the medium supplemented with FBS (data not shown). This experiment provides a viable experimental model for the simulation of the cholestatic microenvironment in vitro.

Bile acid and severe liver injury during cholestasis can trigger an innate immune response $(29,38)$. Secondary infection aggravates cholestasis-induced liver injury and further activates the immune response. Several studies have shown that IL-1 $\beta$ and IL-18 can trigger a specific pro-inflammatory type of cell death during an immune response that may be damaging to various organs (39-41). The accumulation of IL-1 $\beta$ and IL-18 has been shown to aggravate alcohol-mediated and acetaminophen-induced liver hepatotoxicity during liver injury $(15,34,42,43)$. However, TLR9 is able to regulate the accumulation of IL-1 $\beta$ and IL-18 $(15,42)$. The present study revealed that IL-1 $\beta$ and IL-18 accumulated in liver tissue while the inhibition of TLR9 attenuated their accumulation during cholestasis. These findings indicate that IL-1 $\beta$ and IL-18 play a key role in cholestasis-induced hepatotoxicity, which is in line with previous findings.

Several studies have demonstrated that the TLR-mediated innate immune response is associated with liver damage and inflammation. In particular, TLR9 has a pathological role in infection-induced liver injury (44-46), as well as in alcoholor acetaminophen-induced liver injury $(15,34)$. The present study found that HSP90B1 was upregulated in cholestatic liver tissues. A previous study reported the binding affinity of 17-DMAG and HSP90B1 (27). HSP90B1 has been identified as an essential driver for TLR signaling, including TLR9 signaling (17). The present study found that the administration of 17-DMAG decreased IL-1 $\beta$ and IL-18 expression during cholestasis-induced liver injury in vivo and in vitro. Previous studies have also demonstrated that IL-1 $\beta$ and IL-18 facilitate the apoptosis of hepatocytes during liver injury, and that their biogenesis can be regulated by TLR 9 signaling $(15,31,34)$. These findings suggest that 17-DMAG alleviates cholestasis-induced liver injury via the inhibition of HSP90s and TLR9 signaling and the subsequent reduction of IL-1 $\beta$ and IL-18 expression, as demonstrated by the schematic in Fig. 3E.

As a critical chaperone, HSP90 helps to regulate the folding, maturation, stabilization and activation of $>300$ client proteins (47). 17-DMAG is a semi-synthetic HSP90 inhibitor that has several advantages over other geldanamycin derivatives, such as higher water solubility, good bioavailability, reduced metabolism and greater antitumor effects (23). 17-DMAG has been studied in preclinical and clinical trials for certain refractory diseases, including cancer and inflammatory diseases (23). However, some dose-limiting toxicities have been observed during treatment with 17-DMAG, including fatigue, nausea, vomiting, diarrhea and anorexia (48-51). Despite significant improvements, the safety and efficacy of HSP90 inhibitors require further study prior to their clinical translation.

In summary, the results of the present study showed that the HSP90 inhibitor 17-DMAG protected against cholestasis-induced liver injury in rats, via a mechanism involving the reduction of IL-1 $\beta$ and IL-18 expression.

\section{Acknowledgements}

Not applicable.

\section{Funding}

The research was supported by Zhejiang Provincial Natural Science Foundation of China under grant no. LY19H160016, National Natural Science Foundation of China (NSFC; grant no. 81602044), Zhejiang Provincial Public Welfare Technology Application Research Projects (grant no. LGF18H030008) and Research Foundation of Health Bureau of Zhejiang Province (grant nos. 2020RC127, 2019ZD057, 2018KY836 and 2018RC077).

\section{Availability of data and materials}

The datasets used and/or analyzed during the current study are available from the corresponding author on reasonable request.

\section{Authors' contributions}

JY and BL designed the present study. CT, JL, WL, WC and JY performed the experiments. $\mathrm{WZ}$ and $\mathrm{ZZ}$ analyzed the data. CT, JL, JY and BL drafted and revised the paper. JY and BL can authenticate the raw data in this study. All authors read and approved the final manuscript.

\section{Ethics approval and consent to participate}

The present study was approved by the Ethics Committee of Shaoxing People's Hospital (Shaoxing, China).

\section{Patient consent for publication}

Not applicable.

\section{Competing interests}

The authors declare that they have no competing interests.

\section{References}

1. Addley $\mathrm{J}$ and Mitchell RM: Advances in the investigation of obstructive jaundice. Curr Gastroenterol Rep 14: 511-519, 2012.

2. Mosler P: Diagnosis and management of acute cholangitis. Curr Gastroenterol Rep 13: 166-172, 2011.

3. Lazaridis KN and LaRusso NF: The cholangiopathies. Mayo Clin Proc 90: 791-800, 2015.

4. O'Neill S, Ross JA, Wigmore SJ and Harrison EM: The role of heat shock protein 90 in modulating ischemia-reperfusion injury in the kidney. Expert Opin Investig Drugs 21: 1535-1548, 2012.

5. Tukaj S and Wegrzyn G: Anti-Hsp90 therapy in autoimmune and inflammatory diseases: A review of preclinical studies. Cell Stress Chaperones 21: 213-218, 2016.

6. Schopf FH, Biebl MM and Buchner J: The HSP90 chaperone machinery. Nat Rev Mol Cell Biol 18: 345-360, 2017.

7. Wu J, Liu T, Rios Z, Mei Q, Lin X and Cao S: Heat shock proteins and cancer. Trends Pharmacol Sci 38: 226-256, 2017.

8. Usmani SZ, Bona RD, Chiosis G and Li Z: The anti-myeloma activity of a novel purine scaffold HSP90 inhibitor PU-H71 is via inhibition of both HSP90A and HSP90B1. J Hematol Oncol 3: 40, 2010. 
9. Haque A, Alam Q, Alam MZ, Azhar EI, Sait KH, Anfinan N, Mushtaq G, Kamal MA and Rasool M: Current understanding of HSP90 as a novel therapeutic target: An emerging approach for the treatment of cancer. Curr Pharm Des 22: 2947-2959, 2016.

10. Fuhrmann-Stroissnigg H, Ling YY, Zhao J, McGowan SJ, Zhu Y, Brooks RW, Grassi D, Gregg SQ, Stripay JL, Dorronsoro A, et al: Identification of HSP90 inhibitors as a novel class of senolytics. Nat Commun 8: 422, 2017.

11. Ambade A, Catalano D, Lim A and Mandrekar P: Inhibition of heat shock protein (molecular weight $90 \mathrm{kDa}$ ) attenuates proinflammatory cytokines and prevents lipopolysaccharide-induced liver injury in mice. Hepatology 55: 1585-1595, 2012.

12. Ambade A, Catalano D, Lim A, Kopoyan A, Shaffer SA and Mandrekar P: Inhibition of heat shock protein 90 alleviates steatosis and macrophage activation in murine alcoholic liver injury. J Hepatol 61: 903-911, 2014.

13. Medzhitov R: Toll-like receptors and innate immunity. Nat Rev Immunol 1: 135-145, 2001.

14. Khalafalla MG, Woods LT, Camden JM, Khan AA,Limesand KH Petris MJ, Erb L and Weisman GA: P2X7 receptor antagonism prevents IL- $1 \beta$ release from salivary epithelial cells and reduces inflammation in a mouse model of autoimmune exocrinopathy. J Biol Chem 292: 16626-16637, 2017.

15. Teratani T, Tomita K, Suzuki T, Furuhashi H, Irie R, Hida S, Okada Y, Kurihara C, Ebinuma H, Nakamoto N, et al: Free cholesterol accumulation in liver sinusoidal endothelial cells exacerbates acetaminophen hepatotoxicity via TLR9 signaling. J Hepatol 67: 780-790, 2017.

16. Liu B, Yang Y, Qiu Z, Staron M, Hong F, Li Y, Wu S, Li Y, Hao B, Bona R, et al: Folding of Toll-like receptors by the HSP90 paralogue gp96 requires a substrate-specific cochaperone. Nat Commun 1: 79, 2010.

17. Wang J, Grishin AV and Ford HR: Experimental anti-inflammatory drug semapimod inhibits TLR signaling by targeting the TLR chaperone gp96. J Immunol 196: 5130-5137, 2016.

18. Yu J, Zhang W, Qian H, Tang H, Lin W and Lu B: SOCS1 regulates hepatic regenerative response and provides prognostic makers for acute obstructive cholangitis. Sci Rep 7: 9482, 2017.

19. Yang J and Lu B: Establishment of a novel rat model of severe acute cholangitis. Iran J Basic Med Sci 18: 1124-1129, 2015.

20. Livak KJ and Schmittgen TD: Analysis of relative gene expression data using real-time quantitative PCR and the 2(-Delta Delta C(T)) method. Methods 25: 402-408, 2001.

21. Braet F, De Zanger R, Sasaoki T, Baekeland M, Janssens P, Smedsrød B and Wisse E: Assessment of a method of isolation, purification, and cultivation of rat liver sinusoidal endothelial cells. Lab Invest 70: 944-952, 1994.

22. Navaneethan U, Jayanthi V and Mohan P: Pathogenesis of cholangitis in obstructive jaundice-revisited. Minerva Gastroenterol Dietol 57: 97-104, 2011.

23. Mellatyar H, Talaei S, Pilehvar-Soltanahmadi Y, Barzegar A, Akbarzadeh A, Shahabi A, Barekati-Mowahed $M$ and Zarghami N: Targeted cancer therapy through 17-DMAG as an Hsp90 inhibitor: Overview and current state of the art. Biomed Pharmacother 102: 608-617, 2018.

24. Holzbeierlein JM, Windsperger A and Vielhauer G: Hsp90: A drug target? Curr Oncol Rep 12: 95-101, 2010.

25. Kim HR, Kang HS and Kim HD: Geldanamycin induces heat shock protein expression through activation of HSF1 in K562 erythroleukemic cells. IUBMB Life 48: 429-433, 1999.

26. Wang YL, Shen HH, Cheng PY, Chu YJ, Hwang HR, Lam KK and Lee YM: 17-DMAG, an HSP90 inhibitor, ameliorates multiple organ dysfunction syndrome via induction of HSP70 in endotoxemic rats. PLoS One 11: e0155583, 2016.

27. Ge J, Normant E, Porter JR, Ali JA, Dembski MS, Gao Y, Georges AT, Grenier L, Pak RH, Patterson J, et al: Design, synthesis, and biological evaluation of hydroquinone derivatives of 17-amino-17-demethoxygeldanamycin as potent, water-soluble inhibitors of Hsp90. J Med Chem 49: 4606-4615, 2006.

28. Vaughan AT, Betti CJ and Villalobos MJ: Surviving apoptosis. Apoptosis 7: 173-177, 2002

29. Li M, Cai SY and Boyer JL: Mechanisms of bile acid mediated inflammation in the liver. Mol Aspects Med 56: 45-53, 2017.

30. Tanasescu C: Correlation between cholestasis and infection. Rom J Gastroenterol 13: 23-27, 2004

31. Luan J and Ju D: Inflammasome: A double-edged sword in liver diseases. Front Immunol 9: 2201, 2018.

32. Shetty S, Lalor PF and Adams DH: Liver sinusoidal endothelial cells-gatekeepers of hepatic immunity. Nat Rev Gastroenterol Hepatol 15: 555-567, 2018.
33. Cai J, Zhang XJ and Li H: The role of innate immune cells in nonalcoholic steatohepatitis. Hepatology 70: 1026-1037, 2019

34. Roh YS, Zhang B, Loomba R and Seki E: TLR2 and TLR9 contribute to alcohol-mediated liver injury through induction of CXCL1 and neutrophil infiltration. Am J Physiol Gastrointest Liver Physiol 309: G30-G41, 2015.

35. Madrigal-Matute J, Fernandez-Garcia CE, Gomez-Guerrero C, Lopez-Franco O, Muñoz-Garcia B, Egido J, Blanco-Colio LM and Martin-Ventura JL: HSP90 inhibition by 17-DMAG attenuates oxidative stress in experimental atherosclerosis. Cardiovasc Res 95: 116-123, 2012.

36. Qi J,Liu Y, Yang P, Chen T, Liu XZ, Yin Y,Zhang J and Wang F: Heat shock protein 90 inhibition by 17-dimethylaminoethylamino-17-demethoxygeldanamycin protects blood-brain barrier integrity in cerebral ischemic stroke. Am J Transl Res 7: 1826-1837, 2015.

37. Leung AM, Redlak MJ and Miller TA: Role of heat shock proteins in oxygen radical-induced gastric apoptosis. J Surg Res 193: 135-144, 2015

38. Arab JP, Cabrera D and Arrese M: Bile acids in cholestasis and its treatment. Ann Hepatol 16 (Suppl 1: S3-S105): S53-S57, 2017.

39. Liu C, Chen J, Liu B, Yuan S, Shou D, Wen L, Wu X and Gong W: Role of IL-18 in transplant biology. Eur Cytokine Netw 29: 48-51, 2018.

40. Bortolotti P, Faure E and Kipnis E: Inflammasomes in tissue damages and immune disorders after trauma. Front Immunol 9: 1900,2018

41. Mende R, Vincent FB, Kandane-Rathnayake R, Koelmeyer R, Lin E, Chang J, Hoi AY, Morand EF, Harris J and Lang T: Analysis of serum interleukin (IL)-1 $\beta$ and IL-18 in systemic lupus erythematosus. Front Immunol 9: 1250, 2018.

42. Imaeda AB, Watanabe A, Sohail MA, Mahmood S Mohamadnejad M, Sutterwala FS, Flavell RA and Mehal WZ: Acetaminophen-induced hepatotoxicity in mice is dependent on Tlr9 and the Nalp3 inflammasome. J Clin Invest 119: 305-314, 2009.

43. Khanova E, Wu R, Wang W, Yan R, Chen Y, French SW, Llorente C, Pan SQ, Yang Q, Li Y, et al: Pyroptosis by caspase11/4-gasdermin-D pathway in alcoholic hepatitis in mice and patients. Hepatology 67: 1737-1753, 2018

44. Kader M, Alaoui-El-Azher M, Vorhauer J, Kode BB, Wells JZ, Stolz D, Michalopoulos G, Wells A, Scott M and Ismail N: MyD88-dependent inflammasome activation and autophagy inhibition contributes to Ehrlichia-induced liver injury and toxic shock. PLoS Pathog 13: e1006644, 2017.

45. Hackstein CP, Assmus LM, Welz M, Klein S, Schwandt T, Schultze J, Förster I, Gondorf F, Beyer M, Kroy D, et al: Gut microbial translocation corrupts myeloid cell function to control bacterial infection during liver cirrhosis. Gut 66: 507-518, 2017.

46. Mridha AR, Haczeyni F, Yeh MM, Haigh WG, Ioannou GN, Barn V, Ajamieh H, Adams L, Hamdorf JM, Teoh NC and Farrell GC: TLR9 is up-regulated in human and murine NASH: Pivotal role in inflammatory recruitment and cell survival. Clin Sci (Lond) 131: 2145-2159, 2017.

47. Li L, Wang L, You QD and Xu XL: Heat shock protein 90 inhibitors: An update on achievements, challenges, and future directions. J Med Chem 63: 1798-1822, 2020.

48. Ramanathan RK, Egorin MJ, Erlichman C, Remick SC, Ramalingam SS, Naret C, Holleran JL, TenEyck CJ, Ivy SP and Belani CP: Phase I pharmacokinetic and pharmacodynamic study of 17-dimethylaminoethylamino-17-demethoxygeldanamycin, an inhibitor of heat-shock protein 90 , in patients with advanced solid tumors. J Clin Oncol 28: 1520-1526, 2010.

49. Lancet JE, Gojo I, Burton M, Quinn M, Tighe SM, Kersey K, Zhong Z, Albitar MX, Bhalla K, Hannah AL and Baer MR: Phase I study of the heat shock protein 90 inhibitor alvespimycin (KOS-1022, 17-DMAG) administered intravenously twice weekly to patients with acute myeloid leukemia. Leukemia 24: 699-705, 2010.

50. Kummar S, Gutierrez ME, Gardner ER, Chen X, Figg WD, Zajac-Kaye M, Chen M, Steinberg SM, MuirCA, Yancey MA, et al: Phase I trial of 17-dimethylaminoethylamino-17-demethoxygeldanamycin (17-DMAG), a heat shock protein inhibitor, administered twice weekly in patients with advanced malignancies. Eur J Cancer 46: 340-347, 2010.

51. Jhaveri K, Miller K, Rosen L, Schneider B, Chap L, Hannah A, Zhong Z, Ma W, Hudis C and Modi S: A phase I dose-escalation trial of trastuzumab and alvespimycin hydrochloride (KOS-1022; 17 DMAG) in the treatment of advanced solid tumors. Clin Cancer Res 18: 5090-5098, 2012.

This work is licensed under a Creative Commons Attribution-NonCommercial-NoDerivatives 4.0 International (CC BY-NC-ND 4.0) License. 